\title{
Structure-assisted Design of Universal Vaccines and Therapeutics against Influenza Virus
}

Ian A. Wilson,

Department of Integrative Structural and Computational Biology and the Skaggs Institute for Chemical Biology, The Scripps Research Institute, La Jolla, CA 92037, USA.

Email:wilson@scripps.edu

Influenza virus remains a constant threat to global health. The 1918 H1N1 pandemic caused around 50 million deaths worldwide and up to $30-50 \%$ mortality has been reported for recent emerging viruses, such as H5N1 and H7N9, in those hospitalized. Therefore, there is an urgent need to design a much more effective vaccine and therapies to protect against the multiple subtypes and types of influenza virus. Until relatively recently, it was thought that antibodies to influenza virus were strain-specific and could protect only against highly related strains within the same subtype. However, since 2008, many potent human antibodies have been isolated that target the hemagglutinin glycoprotein (HA) and are much broader in their neutralization of influenza virus. We have determined crystal structures of a number of these broadly neutralizing human antibodies (bnAbs) and shown that they bind the highly conserved functional sites on the HA: the stem (fusion domain) and receptor binding site. These structures have revealed common motifs for HA recognition despite different antibody origins and germlines. This information has been utilized in design of vaccine immunogens as well as small proteins, peptides and small molecules as potential therapeutics that target these HA functional sites. Thus, structural and functional characterization of human bnAbs against the HA have provided exciting new opportunities for design of novel vaccines and therapeutics that afford greater protection against influenza virus. 